\title{
Comparison of effectiveness of Salvadora persica whitening toothpaste with commercial whitening toothpaste at removing stain
}

\author{
Erlina Sih Mahanani, Erry Mochamad Arief, Samantha Victoria Samuel
}

School of Dental Sciences, University Sains Malaysia

\begin{abstract}
The miswak, a traditional chewing stick for cleaning teeth, is made from the plant Salvadora persica. It contains wide range of healthy components in maintaining good oral hygiene. The objective of this study is to determine the effectiveness of whitening toothpaste containing Salvadora persica extract at removing tea and chlorhexidine stain by comparing with one of commercially available whitening toothpaste and drinking water as control group. A randomized experimental study was done. The standard method cycled clear acrylic specimens through artificial saliva, $0.2 \%$ chlorhexidine and tea on the hour 8 times per day and stain was measured using a spectrophotometer to achieve baseline reading. Test interventions were 2 whitening toothpastes; whitening toothpaste containing Salvadora persica extract (group A), commercial whitening toothpaste (group B) and drinking water as control group (group C). Treatment interventions were done at 09:00 and 16:00 by exposing the specimens to slurries of each treatment for 2 minutes. The specimens remained in the artificial saliva between each cycles day and night. At the end of the day, the stain was assessed by spectrophotometer and Lobene Stain Index. The comparison of total stain removal assessed by spectrophotometer on day 5 between all groups was significant $(p=0.007)$. The median of total stain removal and visual assessment recording using Lobene stain index showed Salvadora persica whitening toothpaste scored more reduction of staining followed by commercial whitening toothpaste and then control group. As conclusion, the whitening toothpaste containing Salvadora persica is more effective at removing tea and chlorhexidine stain.
\end{abstract}

Key words: Salvadora persica, whitening toothpaste, staining

\section{INTRODUCTION}

Dental staining can occur on the external surface of the tooth (extrinsic staining) or within the tooth substance (intrinsic staining). The extrinsic stain may be removed by tooth brushing, scaling or prophylaxis but the intrinsic stain cannot be removed.

Clinical and in vitro studies have implicated dietary components as major aetiological factors in staining of teeth and acrylic materials associated with chlorhexidine. ${ }^{1}$ The combination of tea and chlorhexidine appears to be a very potent staining factor for extrinsic stain. ${ }^{2}$ The interaction of chlorhexidine with dietary chromogens to cause extrinsic dental staining has been exploited in vitro and in vivo to study tooth discoloration and its control. ${ }^{3}$ Study by Leard and Addy ${ }^{4}$ has

Correspondence author: Erlina Sih Mahanani, School of Dental Sciences, Universiti Sains Malaysia, 16150 Kubang Kerian, Kelantan, Malaysia 
indicated that tea and coffee contribute to dental and tongue staining associated with chlorhexidine mouthrinses.

Mouthwash containing chlorhexidine causes superficial black and brown staining of the teeth. ${ }^{5,6}$ The staining is enhanced in the presence of tea and coffee. It may be related to the precipitation of chromogenic dietary factors to the teeth and mucous membranes. ${ }^{5}$ It is probable that the associated cationic group attaches chlorhexidine to the tooth, while the other cationic group producing the bacteriocidal effect can attach the dietary factors, such as gallic acid derivatives (polyphenols) found in foods and beverages such as tea and coffee and tannins, from wine to the molecule and hence to the tooth surface. ${ }^{4,6}$ It was found that chlorhexidine absorbed to the pellicle and caused a modification of the pellicle properties, leading to subsequent increase in adsorption of salivary and black tea components. This increase in adsorption resulted in a concomitant increase in the stain. ${ }^{7}$

The miswak, a traditional chewing stick for cleaning teeth, is made from the plant Salvadora persica. ${ }^{8}$ The popularity and availability of this chewing stick in the Asia, Middle East, and Africa make them a commonly used oral hygiene tool in those societies. ${ }^{9}$ It has been shown to contain trimethylamine, salvadorine, chloride, fluoride, silica, sulphur, mustard oil, vitamin C, resins and traces of tannins, saponins, flavonids and sterol. ${ }^{10,11}$ Hatab $^{12}$ analyzed the sticks from Salvadora persica for their soluble and total content of flouride, calcium, phosphorus and silica. There was a substantial amount of silica in the ashes of miswak. It appears that miswak is probably not particularly active against caries through its fluoride content, but it does act as a brush for removing dental plaque and polishing the teeth.

As the dental aesthetics are highly concerned nowadays, there has been increase in the demand from consumers and patients for products that whiten teeth. A number of whitening toothpastes have been formulated to inhibit or remove extrinsic staining of teeth. ${ }^{13}$ Some of the manufacturers had produced whitening toothpaste containing Salvadora persica extract. Salvadora persica is a common source to be used as miswak (chewing stick). For religious and cultural reasons, miswak use is firmly established and widespread in most of Muslim countries. It has been shown to contain trimethylamine, salvadorine, chloride, fluoride, silica, sulphur, mustard oil, vitamin C, resins and traces of tannins, saponins, flavonids and sterol. ${ }^{11,14}$ Many studies have demonstrated the antiplaque, antiperiopathic, anticaries and antibacterial effect of it. However, there is no study reported yet regarding its efficacy at removing tea and chlorhexidine stain in comparing with any commercially available whitening toothpaste. Therefore, this study was conducted to determine the effectiveness of whitening toothpaste containing Salvadora persica compared to one of commercial whitening toothpaste and drinking water as control group at removing tea and chlorhexidine stain.

\section{MATERIALS AND METHODS}

Sixty acrylic blocks measuring $4.5 \times 1 \times 1 \mathrm{~cm}$ were cut and polished to fit the specimen chamber of a UV/visible spectrophotometer. Firstly, all specimens were stained until reached the baseline optical reading of spectrophotometer (1.500-1.700). Then the specimens were randomly divided into 3 groups according to the respective treatment regimen.

The staining was produced by cycling specimens through artificial saliva for 2 minutes, a $0.2 \%$ chlorhexidine mouth rinse for 2 minutes and a tea solution for 30 minutes. The tea solution was produced by mixing 5 teabags of tea in 1 litre of water at $50^{\circ} \mathrm{C}$ temperature. The whole staining process was repeated for 8 times a day. The specimens were rinsed with distilled water after each component of cycle was done. Baseline optical density readings for range between 1.500 1.700 were taken for all specimens by using spectrophotometer at lambda maximum of 700 $\mathrm{nm}$. The staining of specimens were also graded visually using the intensity score of the Lobene stain index $(0=$ no stain, $1=$ light stain, $2=$ moderate stain, $3=$ heavy stain). ${ }^{15}$

For both toothpastes, standard slurry was prepared by thoroughly mixing $5 \mathrm{gm}$ toothpaste in $200 \mathrm{~mL}$ distilled water using rotary mixer. This preparation was done to exclude any activity because of presence of abrasives in either toothpaste when applied to toothbrush. The specimens were exposed to the slurries for 2 
minutes. The treatment regimens were whitening toothpaste containing Salvadora persica (Safi kayu sugi whitening toothpaste) as Group A. Gommercially available whitening toothpaste we used Colgate Herbal White (Group B) and drinking water as control group (Group C). The interventions of whitening were done at 09:00 am and 16:00 pm. After the second intervention, the specimens were rinsed briefly with distilled water, bench dried and the optical density read on the spectrophotometer and visual assessment recording using Lobene stain index. ${ }^{15}$ The optical density reading was done for 3 times on the same surface of each specimen. The visual assessment was done by one investigator. All specimens were left in the artificial saliva until the next cycle in the next day. The experiment was done for 5 days.

\section{RESULTS}

The difference of median total stain removal from spectrophotometer reading among groups after 5 days of interventions was significant $(p=0.007)$ is shown in Table 1 . Salvadora persica (group A) shows the highest median (IQR), followed by commercial whitening toothpaste (group B) and control group is the lowest.

The visual assessment recording using Lobene stain index for all groups before the treatment interventions scored grade 3 (heavy stain). On day 5, the result from Table 2 and Figure 1 shows that group $C$ has the highest percentagescoring grade 3 while group $B$ was less and none of group A scored grade 3. It means that group A (Salvadora persica whitening toothpaste) has highest ability to remove the stain.

\section{DISCUSSION}

This model of stain formation or stain removal has been used for many years in dental research. Optically clear acrylic has been used since it is a common dental material which appears to stain in a similar manner to enamel. Besides, it also has the advantage of allowing accurate optical density readings to be obtained. ${ }^{14}$ Using the same brand of tea and chlorhexidine standardize the staining effect on the specimens. The specimens

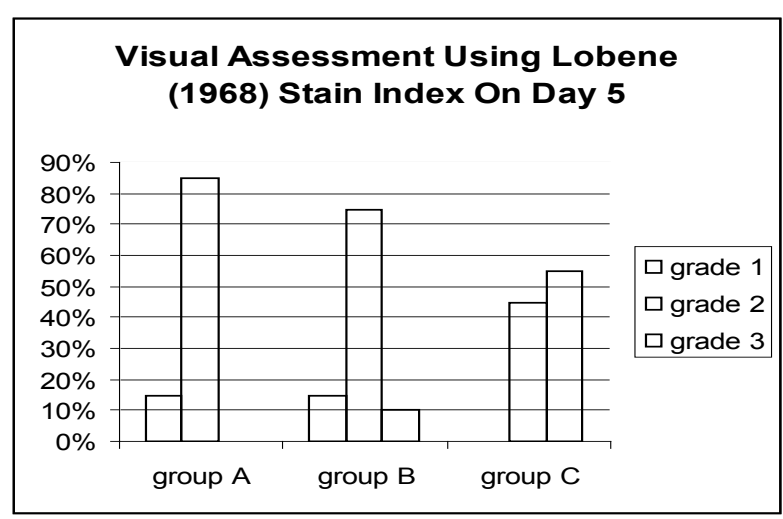

Figure 1. Percentage of specimens in each groups scored for each grade on day 5 .

Table 1. Comparing numerical variables between 3 groups on day 5

\begin{tabular}{cccccc}
\hline Variable & Group & $\mathrm{n}$ & Median (IQR) & Z Statistics (df) & P value $^{\mathrm{a}}$ \\
\hline Total stain removal & A & 20 & $0.2795(0.45)$ & & \\
& B & 20 & $0.2525(0.24)$ & 9.935 & 0.007 \\
& C & 20 & $0.1340(0.15)$ & $(2)$ & \\
\hline
\end{tabular}

${ }^{a}$ Kruskall-Wallis test

Table 1. Frequency distribution of grades using Lobene stain index on day 5

\begin{tabular}{|c|c|c|c|c|c|}
\hline \multirow[b]{2}{*}{ Group } & \multicolumn{4}{|c|}{ Grade } & \multirow{2}{*}{$\begin{array}{l}\text { Total } \\
\text { n (\%) }\end{array}$} \\
\hline & $\begin{array}{c}3 \\
n(\%)\end{array}$ & $\begin{array}{c}2 \\
\text { n (\%) }\end{array}$ & $\begin{array}{c}1 \\
\text { n (\%) }\end{array}$ & $\begin{array}{c}0 \\
\text { n (\%) }\end{array}$ & \\
\hline A & & $17(85)$ & $3(15)$ & & $20(100)$ \\
\hline B & $2(10)$ & $15(75)$ & $3(15)$ & & $20(100)$ \\
\hline C & $11(55)$ & $9(45)$ & & & $20(100)$ \\
\hline Total & 13(21.7) & $41(68.3)$ & $6(10)$ & & $60(100)$ \\
\hline
\end{tabular}

Note: $0=$ No stain, $1=$ light stain, $2=$ moderate stain, 3 heavy stain 
were treated with saliva to form an initial pellicle layer and facilitate the uptake of the stain. ${ }^{16}$ The artificial saliva was used as replacement for human saliva to create standard saliva used throughout this study. To get the human saliva from the same person was time consuming and it was afraid that any changes of the saliva properties during period the study may affect the results of study. The artificial saliva that has been used was produced by Pharmacological Laboratory of HUSM. It contains syrup, lemon, spirit and glycerol with pH 6.5.

In this study, the specimens were stained with tea solution at $50^{\circ} \mathrm{C}$. Tea temperature was significantly influenced staining and the maximum staining effect of tea was at $50^{\circ} \mathrm{C}$. Chlorhexidine temperature did not influence staining. Spectrophotometer reading was highly sensitive since it can detect the difference of stain removal in within 5 day. Therefore, the study could be done in 5 days. The interventions were done twice a day at 09:00 and 16:00 to fit more closely with morning and evening tooth brushing. ${ }^{3}$

Whitening toothpaste with Salvadora persica extract (Safi Kayu sugi whitening toothpaste) contains calcium carbonate, water, sorbitol, glycerine, silica, sodium lauryl sulphate, cellulose gum, flavour, sodium benzoate, sodium saccharin, Salvadora persica and sodium monofluorophosphate. The Colgate Herbal Whitening toothpaste (group B) contains calcium carbonate, water, sorbitol, hydrated silica, sodium lauryl sulphate, flavour, sodium monofluorophospahate, sodium carbonate, cellulose gum, sodium saccharin, carrageenan, xanthan gum, methylparaben, propylparaben, Melissa officinalis extract, Lonicera caprifolium extract, Citrus medica limonium oil and pearl powder. Abrasive agents in toothpaste are believed to remove stain on teeth. The abrasive agents in both toothpastes that were used in this study were calcium carbonate and silica. Another abrasive agent in the commercial whitening toothpaste was sodium carbonate, pearl powder. However, the abrasives effects were excluded in this study as the specimens were placed in the toothpaste slurries during the treatment interventions. Therefore, the whitening effect of these toothpastes was most probably due to the chemical effect.

The combination of herbal extracts (Melissa officinalis and Lonicera caprifolium, Citrus me- dica limonium oil) and abrasive agents in the commercial whitening toothpaste are claimed by the manufacturer to be involved in staining removal. Addition of Salvadora persica in the whitening toothpaste gives significant effect at removing tea and chlorhexidine stain. The results of this study showed that the whitening toothpaste containing Salvadora persica is more effective at removing tea and chlorhexidine stain compared to the commercially available whitening toothpaste. According to Darout ${ }^{17}$, release of beneficial substances in Salvadora persica have been suggested to contribute to the cleaning efficacy of miswak. High concentration of chloride in Salvadora persica was suggested influencing the result as it was reported to help in removing stains from teeth. ${ }^{9}$ Further research may be required to study regarding the chemical agents in Salvadora persica that probably cause stains removal.

From the study, drinking water that has been used as control group also showed some removal of tea and chlorhexidine stain but it was minimal. Therefore, rinsing with water may remove tea and chlorhexidine stain to some minimal extension.

\section{CONCLUSION}

The whitening toothpaste containing Salvadora persica is more effective at removing tea and chlorhexidine stain compared to one of commercially available whitening toothpaste. The ability of this toothpaste due to the chemical effect cause the intervention is remained in the toothpaste slurries without brushing the surface of specimens. Further study need to investigate the mechanical effectiveness of this toothpaste.

\section{REFERENCES}

1. Addy M, Moran J. The formation of stain on acrylic surfaces by the interaction of cationic antiseptic mouthwashes and tea. J Bioned Mater Res 1984;18(6):631-41.

2. Carpenter GH, Pramanik R, Proctor GB. An in vitro model of chlorhexidine-induced tooth staining. J Periodontal Res 2005;40(3):22530.

3. Pontefract $\mathrm{H}$, Courthey $M$, Smith $\mathrm{S}$, Newcombe RG, Addy M. Development of methods to enhance extrinsic tooth discoloration for 
comparison of toothpastes. 1. Studies in vitro. J Clin Periodontol 2004;31(1):1-6.

4. Leard A, Addy M. The propensity of different brands of tea and coffee to cause staining asscociated with chlorhexidine. J Clin Periodontol 1997;24(2):115-8.

5. Addy M, Moran J, Griffiths A, Wills-Wood NJ. Extrinsic tooth discolouration by metals and chlorhexidine. I: Surface protein denturation or diet precipitation? Br Dent J 1985;159:331-4.

6. Eley BM. Antibacterial agents in the control of supragingival plaque a review. Br Dent $\mathrm{J}$ 1999;186:286-96.

7. Joiner A. Review of the extrinsic stain removal and enamel/dentine abrasion by a calcium carbonate and perlite containing whitening toothpaste. Int Dent J 2006; 56(4):175-80.

8. Al-Otaibi $M, A l$ Harthy $M$, Gustafsson A, Johansson A, Claesson R, Angmar-Mansson B. Subginggival plaque microbiota in Saudi Arabians alter use of miswak chewing stick and toothbrush. J Clin Periodontol 2004;31:104853.

9. Almas K, Al-Lafi T. The natural toothbrush. World Health Forum 1995;16:206-10.

10. Farooqi MIH, Srivastana JG. The toothbrush tree (Salvadora persica). J Crude Drug Res 1968;8:1297-9.

11. Ezmirly JT, Cheng JC, Wison SR. Saudi Arabian medical plants Salvadora persica. Planta Medica 1978;35:191-2.

12. Hattab FN. Meswak: the natural toothbrush. J Clin Dent 1997;8(5):125-9.

13. Sharif N, MacDonald E, Hughes J, Newcombe RG, Addy $M$. The chemical stain removal properties of 'whitening' toothpaste products: studies in vitro. British Dent J 2000;188(11):620-24.

14. Elvin-Lewis $M$. The therapeutic potential of plants used in dental folk medicine. Odonstomatol Trop 1982;3:107-17.

15. Lobene RR. Effect of dentifrices on tooth stains with controlled brushing. J Am Dent Assoc 1968;77:849-55.

16. Jagger DC, Al-Akhazam L, Harrison A, Rees JS. The effectiveness of seven dentures cleansers on tea stains removal from PMMA acrylic resin. Int J Prosthodont 2002;15(6):549-52.

17. Darout IA, Albandar JM, Skaug N, Ali RW. Salivary microbiota levels in relation to periodontal status, experience of caries and miswak use in Sudanesce adults. J Clin Periodontol 2002;29(5):411-20. 